\title{
Outage severity analysis and RAM evaluation of Italian overhead transmission lines from a regional perspective
}

\author{
Mohamed Khalil, Christian Laurano, Giacomo Leone, Michele Zanoni
}

DEIB, Politecnico di Milano, Piazza Leonardo da Vinci 32, 20133 Milano, Italy

\begin{abstract}
Power transmission lines represent the core of the High Voltage Network since they are responsible for the transport of the electrical energy from the generation power plants to the electrical substations. In this paper, an analysis of the outages occurred to the Italian Overhead Transmission Lines (OHTLs) from 2008 to 2015 is carried out. A new simple and effective reliability index, namely the Severity Factor, is introduced with the aim to drive the prioritization of the failure causes and the enhancement of the maintenance activities. The analysis has been performed focusing on the geographical distribution of the OHTLs. For each analyzed region, the voltage levels more prone to failure have been determined. The proposed methodology, thanks to the introduction of the Severity Factor, is a useful and effective tool for the identification of the transmission network criticalities and the enhancement of the related maintenance activities. Finally, an evaluation of the reliability, availability, safety and maintainability (RAMS) of the Italian OHTL network has been performed from a regional point of view.
\end{abstract}

\section{Section: RESEARCH PAPER}

Keywords: dependability; hazard rate; multi-regional overhead transmission lines analysis; RAMS analysis; Severity Factor

Citation: Mohamed Khalil, Christian Laurano, Giacomo Leone, Michele Zanoni, Outage severity analysis and RAM evaluation of Italian overhead transmission lines from a regional perspective, Acta IMEKO, vol. 5, no. 4, article 11, December 2016, identifier: IMEKO-ACTA-05 (2016)-04-11

Section Editor: Lorenzo Ciani, University of Florence, Italy

Received September 27, 2016; In final form December 6, 2016; Published December 2016

Copyright: (C) 2016 IMEKO. This is an open-access article distributed under the terms of the Creative Commons Attribution 3.0 License, which permits unrestricted use, distribution, and reproduction in any medium, provided the original author and source are credited

Corresponding author: Michele Zanoni, e-mail: michele.zanoni@polimi.it

\section{INTRODUCTION}

Transmission networks represent the core of the electrical system and their failure implies a waste of energy and significant financial losses [1], [2]. Consequently, the aim of the Transmission System Operators (TSO) is to increase the network maintainability and plan its development in order to minimize outages occurrence and make the grid robust to failures preventing serious consequences on the loads.

Overhead Transmission Lines (OHTL) are definitely the most important elements in the High Voltage (HV) network, since they are responsible for the transport of the electrical energy from the generating plants to the loads. It follows that industries and facilities give a great attention to their reliability [3]-[7]. At the same time, however, from a study conducted by the same authors of this paper in [8]-[9], it results that OHTLs are still the most critical components from both number of failures and the corresponding interrupted power points of view. The analysis presented in [8]-[9] were based on the data published by TERNA, the Italian TSO, about the outages of the Italian OHTLs in the period from 2008 to 2014.
In this paper, a new analysis is performed. In particular, much emphasis is put on the geographical distribution of the OHTLs and the related operating voltage level in order to investigate the effect of local factors such as environmental conditions or the regional maintenance strategy on the network reliability. Further, a longer period is surveyed, from 2008 to 2015, during which 12594 outages were registered, with a total power interrupted of 81379 MW. The tools exploited for the outage analysis are the Severity Factor (SF), already introduced by the the same authors in [8]-[9], and the Reliability, Availability, Maintainability (RAM) Analysis.

The paper is structured as follows: in Section 2, a general overview of the Italian TSO TERNA is provided. In Section 3, a brief overview on the transmission systems reliability metrics available in literature is provided. In Section 4, the Severity Factor is introduced and the differences with respect to the traditional metrics are highlighted. The SF is then applied in Section 4 where the outage data analysis is performed. In Section 5, a different analysis based on the RAM technique is carried out. In particular, it is performed independently for each 
OHTLs voltage level and a comparison of the values of Reliability, Availability and Maintainability obtained for the different regions is given. Finally, the contribution ends in Section 6 where the conclusions are provided.

\section{ITALIAN NETWORK OVERVIEW}

Italian network operator, TERNA, is one of the most important in Europe in terms of lines extent (63,900 km of high voltage lines), power generation $(120 \mathrm{GW})$ and energy flowing (300 billion kWh per year). Until 2009, the Italian transmission network was divided in two parts: RTN, property of TERNA, and Telat, property of Enel, the Italian national agency for the electrical energy. Since 2009, however, the grid has been unified under the control of TERNA.

As result of its transparency policy, TERNA yearly publishes an annual report about the outage events occurred in its transmission network [10]. In particular, for each failure event the following data are provided: identification number of the outage, event location, date and time of occurrence, damaged component, voltage level, outage category, kind of interruption (transient, short or long), grid configuration, amount of interrupted power and time to repair.

\subsection{Regional partition}

In order to make a uniform division of the Italian territory, TERNA identifies in its reports eight macro regions (MRs), different from the political regional division of the country. In Table 1 the eight MRs, along with the corresponding Italian regions, are presented.

\subsection{Voltage subpopulations}

For an efficient statistical analysis of the OHTLs outages data, it is useful to consider, besides the geographical partition of the failures, also the voltage level at which they occur. It is reasonable, indeed, to expect that the predominant outage causes for a transmission line may depend on its rated voltage. The classification of the voltage ratings adopted by TERNA is the following: $380 \mathrm{kV}, 220 \mathrm{kV}, 150 \mathrm{kV}, 132 \mathrm{kV}$ and less than $100 \mathrm{kV}$.

In Table 2, the OHTLs territorial distribution for each voltage subpopulation is reported. Such data have been obtained from the TERNA report of 2013. As the surveyed period, ranging from 2008 to 2015, is limited, however, the eventual grid expansions that may have occurred in such years are assumed negligible with respect to the total network extent, hence the reported values can be considered an accurate estimation of OHTLs distribution.

Table 1. TERNA geographical division.

\begin{tabular}{cc}
\hline MR & Italian regions \\
\hline CA (Cagliari) & Sardegna \\
FI (Firenze) & Part of Emilia Romagna - Toscana \\
MI (Milano) & Lombardia - Part of Emilia Romagna \\
NA (Napoli) & Campania - Puglia - Basilicata - Calabria \\
PA (Palermo) & Sicilia \\
PD (Padova) & Friuli Venezia Giulia - Veneto - Trentino Alto Adige \\
RM (Roma) & Lazio - Umbria - Abruzzo - Molise - Marche \\
TO (Torino) & Piemonte - Liguria - Valle d'Aosta \\
CA (Cagliari) & Sardegna \\
FI (Firenze) & Part of Emilia Romagna - Toscana \\
MI (Milano) & Lombardia - Part of Emilia Romagna
\end{tabular}

Table 2. Length of OHTLs (in km) per each MR and voltage level.

\begin{tabular}{cccccc}
\hline MR & $\mathbf{3 8 0} \mathbf{~ k V}$ & $\mathbf{2 2 0} \mathbf{~ k V}$ & $\mathbf{1 5 0} \mathbf{~ V V}$ & $\mathbf{1 3 2} \mathbf{~ k V}$ & $<\mathbf{1 0 0} \mathbf{~ V}$ \\
\hline CA & 314.199 & 554.390 & 1995.282 & - & 200.066 \\
FI & 2052.499 & 703.060 & - & 5673.969 & 79.021 \\
MI & 1514.724 & 2176.880 & - & 5669.887 & 46.544 \\
NA & 2675.879 & 1101.710 & 6646.029 & - & 704.217 \\
PA & 252.59 & 1530.090 & 3170.542 & - & 40.980 \\
PD & 782.031 & 2591.993 & - & 5564.712 & 459.177 \\
RM & 1953.383 & 1005.968 & 3626.473 & 5203.620 & 168.582 \\
TO & 1136.753 & 1741.560 & - & 4343.212 & 119.274
\end{tabular}

It must be specified that, as it can be observed in Table 2, for what regards $150 \mathrm{kV}$ and $132 \mathrm{kV}$ voltage levels, the MR Roma is the only one in which both $150 \mathrm{kV}$ and $132 \mathrm{kV}$ OHTLs are present. This observation, along with the fact that $150 \mathrm{kV}$ and $132 \mathrm{kV}$ OHTL networks are subject to similar operating conditions, justifies the choice of the authors to consider in the followings the voltage subpopulations $150 \mathrm{kV}$ and $132 \mathrm{kV}$ as a unique population.

\subsection{Outage categories}

Transmission lines outages can be grouped in two main categories: forced outages and scheduled outages. Forced outages of OHTLs are mainly due to automatic switching operations performed by the protection systems. They are defined by IEEE Std. 493-1990 [11] as outages that cannot be deferred and result in power interruption or loss of service. On the other side, planned and scheduled outages are interruption planned in advance for routine maintenance or equipment inspection. It follows that planned and scheduled outages should be excluded from any statistical study of OHTLs as they do not represent any criticality on the network management, so that, for the sake of brevity, in the following, the term "outages" refers exclusively to "forced outages".

In its annual reports [10] TERNA specifies for each failure event the corresponding outage category. In particular, it distinguishes five different categories, listed in Table 3. Among these, only category 5DP corresponds to scheduled outages, so that the failure events belonging to this category are not taken into account in the statistical analysis proposed in the following of the paper.

\section{TRANSMISSION SYSTEMS RELIABILITY METRICS}

In literature, different metrics for the evaluation of transmission systems reliability and outages impact on the grid are proposed. An important source is represented by the IEEE Standard 1366 [12] published by the Transmission and Distribution Committee with the scope to create indices specifically designed for transmission and distribution systems, based on [13]-[14]. The indices proposed in [12] are widely used by organizations and national operators in the assessment of

Table 3. TERNA geographical division.

\begin{tabular}{cc}
\hline Category & Description \\
\hline $1 C D$ & Lack of resources \\
$2 \mathrm{FM}$ & Unpredictable events \\
$3 \mathrm{CE}$ & External causes \\
$4 \mathrm{AC}$ & Other causes \\
$5 \mathrm{DP}$ & Scheduled maintenance
\end{tabular}


the quality of the provided service. In particular, in its annual quality report TERNA focuses its attention in the following three specific indices:

1) SAIFI (System Average Interruption Frequency Index) which is expressed as:

$$
\text { SAIFI }=\frac{\sum N_{i}}{N_{T}},
$$

where $N_{i}$ the number of customer interrupted for each interruption, and $N_{T}$ the total number of customers. It indicates how often the average customer experiences a sustained interruption (any interruption that lasts more than five minutes) over a predefined period of time.

2) SAIDI (System Average Interruption Duration Index) which indicates the total duration of the interruption for the average customer over a predefined period of time:

$$
\text { SAIDI }=\frac{\sum r_{i} N_{i}}{N_{T}},
$$

where $r_{i}$ is the restoration time for each interruption event.

3) MAIFI (Momentary Average Interruption Frequency Index), which takes into account the frequency of momentary interruptions, defined as the interruption with a restoration time $r_{i}$ lower than 5 minutes. Given $I M_{i}$ as the number of momentary interruptions and $N_{i}$ the number of interrupted customers for each momentary interruption $i$, MAIFI is mathematically defined as:

$$
\text { MAIFI }=\frac{\sum I M_{i} N_{i}}{N_{T}} .
$$

It is immediately observable from their definition that such metrics provide information only about single features of the outages events, such as frequency of interruptions (SAIFI and MAIFI) or interruptions duration (SAIDI). In particular, TERNA computes the value of these indices for each MR, without discriminating about the operating voltage level of the OHTLs. Furthermore, such metrics are evaluated only for failures belonging to category 4AC. From all these considerations it comes the idea of the authors to carry out a more detailed reliability analysis based on the data of all the OHTL forced outages events (i.e. also categories 1CD, 2FM and $3 \mathrm{CE}$ are taken into account) and the proposal of a new reliability index, the Severity Factor, that synthetizes in a unique number all the information related to the criticality of outage events: frequency of occurrence, interruption duration and corresponding interrupted power. Moreover, the analysis is performed focusing not only on the geographical distribution of the OHTLs but also on the operating voltage level, differently than in [10].

\section{SEVERITY FACTOR}

The Severity Factor has been introduced by the authors in [8]-[9], defining it as an index that enables a rapid and effective comparison of the impact of different outage causes for OHTLs at the same voltage level. In particular, the symbol $\mathrm{SF}_{i, j}$ denoted the Severity Factor of a generic outage category $i$ for the OHTLs of the voltage category $j$. It is interesting to highlight that, because of how it is defined, the concept of
Severity Factor can be exploited also for other kinds of failure data analysis. An interesting example, indeed, is the analysis of the distribution of the failures in a given MR among the different voltage levels. In this case, the notation $\mathrm{SF}_{i, j}$ would refer to the severity of the failures occurred at voltage level $i$ for the $j$-th MR. In the following, for the sake of the clearness, with the notation $\mathrm{SF}_{i, j}$, the authors will always refer to the severity of the failures related to category $i$ and affecting the OHTL belonging to the macrocategory $j$. In this paper, the SF is defined with a different analytical expression with respect to [8], where it was defined in an analogous way with respect to the Risk Priority Number (RPN) adopted in the Failure Mode, Effects and Criticality Analysis (FMECA) [15]. In particular, it will be adopted the definition of severity factor SF related to a category $i$ and macrocategory $j$ introduced in [9] and reported in (4) as:

$$
S F_{i, j}=\frac{\gamma_{i} E_{i, j}}{\sum_{n=1}^{N_{j}} \gamma_{n} E_{n, j}},
$$

where $n=1,2, \ldots, N_{j}$ is the index of each category composing the macrocategory $j$ and $E_{i, j}$ is the total not-transmitted energy consequent to outage events affecting category $i$ inside macrocategory $j$. In particular, $E_{i, j}$ can be computed as:

$$
E_{i, j}=\sum_{e=1}^{N_{e}} P_{e} \cdot t_{e},
$$

where $N_{e}$ is the related total number of outage events, and $P_{c}$ and $t_{e}$ are, respectively, the interrupted power and inactivity time caused by each single event.

As for the factor $\gamma$ in (4), it is a weighting factor that takes into account the different OHTLs kilometric extent of each single category inside macrocategory $j$. In particular, given a category $i$ and a macrocategory $j, \gamma_{i}$ is expressed as:

$\gamma_{i}=\frac{L_{j}}{L_{i}}$,

where $L_{i}$ is the length of the OHTLs belonging the category $i$ and $L_{j}$ the total length of the OTHLs related to the macrocategory $j$. If, for example, the objective is to compute the SF for the failures occurred at different voltage levels in the $\operatorname{MR} j, L_{i}$ would refer to the total length of the lines operating at voltage level $i$ in the macro region $j$. The sum of the lines length at different voltage levels would provide the value of $L j$. Putting $L_{i}$ at the denominator allows to give more weights to the outage events related to categories less represented (in terms of length) inside the subset defined by the macrocategory $j$. This makes the comparison of the SFs for the different categories more fair, since it is reasonable to expect that statistically the number of outage events recorded for a given category $i$ is proportional to the length of the related lines, so that $E_{i, j}$ tends to increase with the length.

The new formulation of the SF given in (4) leads to different improvements with respect to the definition provided in [8]. The generic $S F_{i, p}$ indeed, has now a physical meaning since it represents a weighted percentage of not-transmitted energy associated to a category $i$, for a macrocategory $j$. Furthermore, the sum of the SF of the different categories $i$ for a macrocategory $j$ is now equal to 1 by definition. These improvements make the severity factor easier to understand and able to offer a more intuitive representation of the OHTLs 
outages scenario resulting in a useful tool for the maintenance personnel in the coordination of the maintenance activities. Nevertheless, it is important to emphasize how the SF differs from the other indices presented in Section 3 and available in literature as in its definition different features characterizing the criticality of failure events such as failure frequency, interrupted power and interruption duration are involved at the same time. Another issue to take into account is that traditional indices such as SAIFI, SAIDI and MAIFI require the knowledge of number of customers of the network $N_{T}$ and the number of interruptions experienced by each customer $N_{i}$. Very often, these data are difficult to be obtained and may prevent the computation of the indices starting from public reports as the annual quality report published by TERNA [10]. Conversely, the SF requires for its computation the knowledge of only the number of registered outages and the related downtime and interrupted power, which are data commonly provided by the organizations.

\section{OUTAGE DATA ANALYSIS}

Once having defined the new SF, it is possible to carry out a statistical analysis on the OHTL outages. In this paper, two different analyses are proposed. First, for each MR the Severity Factor of OHTLs operating at different voltage levels is evaluated. In the second analysis, instead, the focus moves towards the comparison of the SF for the different MRs given a specific voltage level.

\subsection{Voltage subpopulation analysis}

The aim of this analysis is to study the severity of the outages occurring at the different voltage levels in a given MR. Therefore, following the notation described in Section 4, the MR of interest is indicated with the index $j$, whereas the voltage population is indicated with the index $i$. Analogously, for what concerns the weighting factor $\gamma_{i}, L_{j}$ is the total length of the OTHL belonging to MR $j$, whereas $L_{i}$ represents the total length of the lines of such MR operating at voltage level $i$.

Thanks to the new definition (4) of the Severity Factor, it is possible to carry out a meaningful SF comparison between different MRs. Furthermore, it is possible to compare the SFs obtained for each MR with the results obtained at national level. The results are shown in Figure 1.

It is interesting to observe how the analysis carried out at national level emphasizes the criticality represented by the OHTLs operating at voltages below $100 \mathrm{kV}$ (row IT in Table 4), when compared to other voltage populations. Moving the analysis to a regional level, however, allows to highlight that this is not the case for the MRs Firenze and Napoli, for which the most severe situations are represented, respectively, by the 132

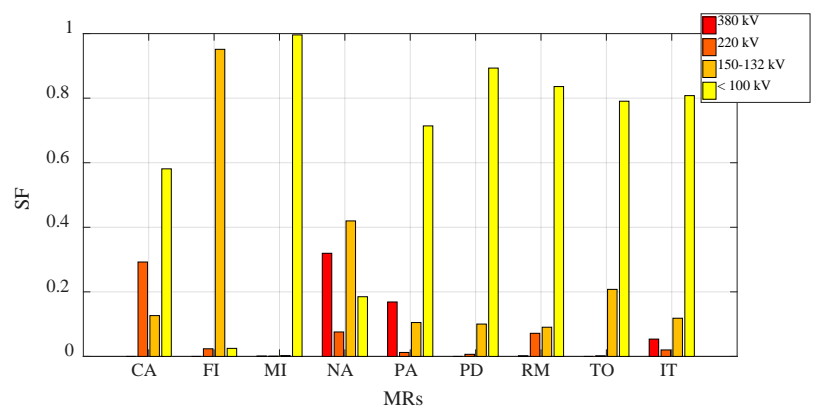

Figure 1. Voltage level SF for different MRs.
Table 4. Severity Factor per each voltage level for a given MR

\begin{tabular}{ccccc}
\hline MR & $\mathbf{3 8 0} \mathbf{~ k V}$ & $\mathbf{2 2 0} \mathbf{~ k V}$ & $\mathbf{1 5 0 - 1 3 2} \mathbf{~ k V}$ & $<\mathbf{1 0 0} \mathbf{~ k}$ \\
\hline CA & 0 & 0.293 & 0.126 & 0.581 \\
FI & 0 & 0.024 & 0.951 & 0.025 \\
MI & 0.001 & 0.001 & 0.002 & 0.996 \\
NA & 0.319 & 0.076 & 0.420 & 0.185 \\
PA & 0.169 & 0.012 & 0.105 & 0.714 \\
PD & 0 & 0.007 & 0.100 & 0.893 \\
RM & 0.002 & 0.072 & 0.090 & 0.836 \\
TO & 0 & 0.002 & 0.208 & 0.790 \\
IT & 0.054 & 0.020 & 0.118 & 0.808
\end{tabular}

$\mathrm{kV}$ grid (SF equal to 0.951 ) and the $380 \mathrm{kV}$ and $150 / 132 \mathrm{kV}$ grids $(0.319$ and 0.420$)$.

Another important result derived from the outage analysis carried out at regional level is the relevant SF computed for the $380 \mathrm{kV}$ population for the MRs Napoli and Palermo, equal to 0.319 and 0.169 respectively. This result highlights the need for more detailed analysis in order to understand the root causes of the outages and reduce the related energy losses. Similar considerations can be made for Firenze where the $132-150 \mathrm{kV}$ grid, having an SF value equal to 0.951 , represents a critical case for which a deep investigation is needed.

It can be concluded that the outages analysis performed at MR level has emphasized that an analysis carried out from a national point of view leads to approximate results that, generally, can be misleading since they are not replicated in the different MRs, because of the different operating conditions and/or maintenance strategies.

\subsection{MRs analysis}

A different point of view is given by the analysis of the SF of the OHTLs among the different MRs for a given voltage level (i.e. considering the regional subdivision as category $i$, and the voltage population as macrocategory $j$ ). In this kind of analysis, for the sake of the computation of the weighting factor $\gamma_{i}$ (the length of the lines at a given voltage level are not the same in each region), $L_{j}$ corresponds to the total length of the OHTLs at a specific voltage level $j$, whereas $L_{i}$ is the length of the lines at that voltage level operating in the $i$-th MR. The achieved results are shown in Figure 2 and reported in Table 5.

The results confirm what already was described in the previous Section 4.1 but allow to highlight also further considerations. It is evident, in fact, that for what concerns the $380 \mathrm{kV}$ voltage grid, Palermo is the most affected MR with an $\mathrm{SF}$ equal to 0.60. This statement was not evident by previous analysis (Figure 1) as Napoli presented a higher SF than

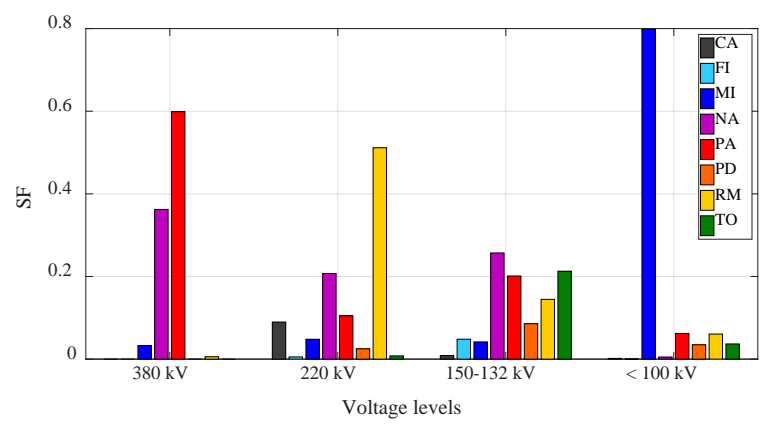

Figure 2. Geographical impact for different voltage levels. 
Table 5. Severity Factor per each MR for a given voltage level.

\begin{tabular}{ccccc}
\hline MR & $\mathbf{3 8 0 ~ k V}$ & $\mathbf{2 2 0} \mathbf{k V}$ & $\mathbf{1 5 0 - 1 3 2 ~ k V}$ & $<\mathbf{1 0 0 ~ k V}$ \\
\hline CA & 0 & 0.090 & 0.009 & 0.002 \\
FI & 0 & 0.005 & 0.048 & 0.000 \\
MI & 0.033 & 0.048 & 0.042 & 0.798 \\
NA & 0.362 & 0.207 & 0.257 & 0.005 \\
PA & 0.599 & 0.105 & 0.201 & 0.062 \\
PD & 0 & 0.025 & 0.086 & 0.035 \\
RM & 0.006 & 0.512 & 0.145 & 0.061 \\
TO & 0 & 0.008 & 0.212 & 0.037
\end{tabular}

Palermo. This difference in the results is justified by the different focus characterizing the two analyses. In particular, the previous analysis focused on the SF of the OHTLs at different voltage levels in the same MR. It can be concluded that Napoli MR is the area for which the $380 \mathrm{kV}$ grid represents the most critical case, but moving at national level it is the MR Palermo that mostly contributes for the severity of the outages occurred at this voltage level.

An analogous situation occurs for the OHTLs operating at less than $100 \mathrm{kV}$. The analysis performed in Section 5.1 showed similar SFs for most of the MRs (Milano, Padova, Roma, Torino had SF between 0.790 and 0.996$)$. These results would let think that such MRs contribute almost equally for the severity of the outages at this voltage level. Results depicted in Figure 2, however, deny this theory as they highlight that Milano MR is by far the most solicited area, presenting an SF equal to 0.80 .

Finally, it can be concluded that both presented analyses are meaningful, providing two complementary points of view on the same problem. On one side the severity of a voltage grid over the failures of a given MR is depicted, whereas on the other the regional severity over a specified voltage subpopulation is considered.

The analysis of the results has highlighted the effectiveness of the proposed SF index for the identification of the most critical voltage levels for the transmission levels at both national and regional levels, resulting as a useful tool for an optimal planning of maintenance activities.

\section{RAM ANALYSIS}

Reliability, Availability and Maintainability (RAM) are parameters that are widely used for system design specifications and as an operational performance indicator for utility assets. Reliability is the probability that a system will be available at a given time. Based on reliability theory [15]-[18], the following definitions are applied:

- Failure rate: during the useful-life phase of a product, failure rate can be defined as the number of random (unscheduled) occurrences of failure of the product to perform its intended function divided by the length of time the product was functioning.

- Maintainability is a property of repairable systems defined as the facility in which a system can be repaired once a malfunction (or failure) is manifested. Maintenance should be performed by personnel having specified skill levels, using prescribed procedures and resources, at each prescribed level of maintenance and repair. A common way to express the maintainability is the reciprocal of the Mean Time To Repair
(MTTR). It is important to note that maintainability is not the same as maintenance.

- Availability is the percentage of time the item is available to perform its required functions. Availability deals with up-time for operations and is a measure of how often the system is alive. It is a function of both Mean Time Between Failures (MTBF) and MTTR. High availability, indeed, does not always mean high reliability, since a system with frequent failures (low reliability, small MTBF) but with very small MTTR (with respect to the MTBF) would be characterized anyway by a high availability.

\subsection{Reliability indicator: Failure rate}

A good indicator of the OHTL reliability is the failure rate. It is calculated based on the following equation (7):

$\lambda=\frac{N}{L \cdot T}$

where $N$ is the total number of outages occurred during the surveyed period $T$ and $L$ is average of the lengths of OHTLs during the surveyed period divided by $100 \mathrm{~km}$.

The failure rates of each voltage level of OHTL for all Italian regions are illustrated in Figure 3. It is depicted that 380 $\mathrm{kV}$ and $220 \mathrm{kV}$ grids are quite robust to outages as the hazard rate computed for this voltage levels is very low for every Italian MR. The hazard rate increases when the focus moves to the $150-132 \mathrm{kV}$ voltage level, with an average value of about 3 failures per year per $100 \mathrm{~km}$, except a small peak related to Palermo MR, which is affected by about 6 failures per year per $100 \mathrm{~km}$. The most critical situation is represented by the voltage level below $100 \mathrm{kV}$ where it is recorded an average of 15 yearly failures per $100 \mathrm{~km}$. The worst case is related to the MRs of Milano and Napoli where the hazard rate is twice as much as in the rest of the country. Looking at the analysis performed in the previous subsection 5.2, this result was predictable for MR Milano, but not for the MR Napoli. This region, indeed, is characterized by a high average number of failures at this voltage level but a very low SF (equal to 0.05), especially if compared to the one of Milano. The reason for this apparent discrepancy has to be found in the weighting factors computed for the definition of the SFs. Such factor, indeed, is very low for the region of Napoli, since it is characterized by a transmission grid operating below $100 \mathrm{kV}$ long about $704 \mathrm{~km}$ (out of a total national extent of the grid at that voltage level equal to 1818 $\mathrm{km})$. Conversely, for the Milano MR a large weighting factor is assigned since the extent of such grid is only $46 \mathrm{~km}$. These considerations highlight the importance to perform, besides the computation of the SF as described in the previous Section 4, also a RAM analysis in order to have a complete set of

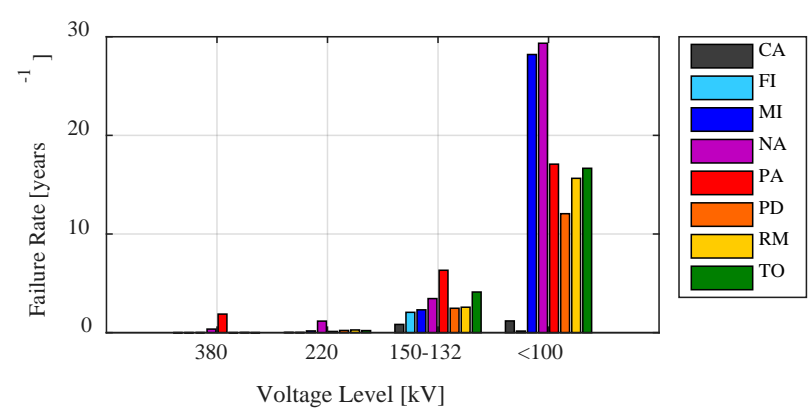

Figure 3. Hazard rate for different voltage subpopulations and macro regions. 
information about the national and regional scenario of the outages affecting the transmission system.

\subsection{Availability}

The availability $A(t)$ is calculated by equation (8) as:

$$
A(t)=\frac{\operatorname{MTBF}(t)}{\operatorname{MTBF}(t)+\operatorname{MTTR}(t)} \%
$$

The availability for OHTLs for various voltage subpopulations in the Italian grid is illustrated in Figure 4.

The availability is concluded to be lower for the OHTLs operating at less than $220 \mathrm{kV}$, even if it never goes below the value $96 \%$. A different scenario is depicted for the $220 \mathrm{kV}$ and $380 \mathrm{kV}$ OHTLs where the availability is very close to 1 in all the regions, except for the case of Torino OTHLs at $220 \mathrm{kV}$ (availability below the $98 \%$ ). This case is very explicative about the importance of the MTTR in the definition of the availability. The Torino $220 \mathrm{kV}$ grid, indeed, presents a very low failure rate equal to about 0.20 failure per year per $100 \mathrm{~km}$, synonym of quite a large $M T B F$ and high reliability. The key parameter in the evaluation of the availability, however, as can be seen from (8), is represented by the ratio $M T T R / M T B F$ since the lower it is the higher is the availability. This ratio has been computed for all the voltage levels and all the Italian MRs and the obtained results are depicted in Figure 5.

It can be observed that for what concerns the $220 \mathrm{kV}$ grid, Torino is characterized by a ratio much higher than other MRs (almost 2 orders of magnitude), resulting in a lower availability. Such a higher MTTR should reflect also in a higher SF, but observing the results reported in Figure 2 and Table 5 this is not the case. The reason lays in the fact that in the computation of the SF a role is played also by the amount of interrupted power at each outage event occurrence, that for the specific

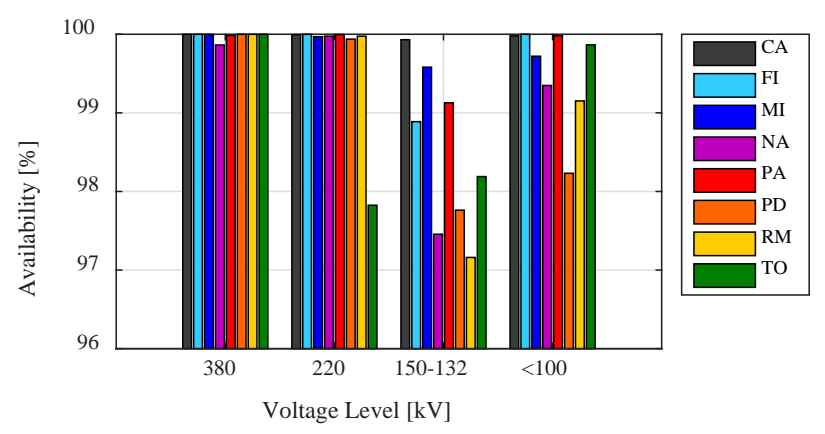

Figure 4. Availability for different voltage subpopulations and macro regions.

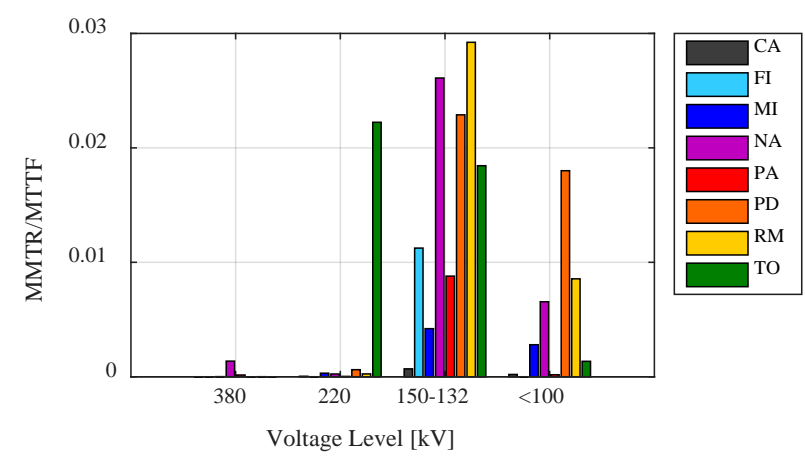

Figure 5. MTTR/MTTF for different voltage subpopulations and macro regions. case of Torino OHTL at $220 \mathrm{kV}$ is quite low. Further, the weighting factor associated to such grid is also low, due to the considerable length of the considered system.

\subsection{Maintainability evaluation}

Different environmental conditions and major load classes according to the main activities in each zone characterize the considered geographical MRs. Some zones are mainly agricultural, while others are of composite load classes. Therefore, maintainability for various voltage subpopulations differs from one zone to another. Maintainability is here expressed as the reciprocal of the Mean Time To Repair (MTTR). The results obtained from the computation of the maintainability of the OHTLs operating at different voltage levels and different MRs are reported in Figure 6. For OHTLs operating at $380 \mathrm{kV}$ in the MRs Cagliari, Firenze, Padova and Torino, the maintainability is not definable as the related MTTR is equal to zero, since no outages were registered during the surveyed period. In general, it seems that it is not possible to denote a characteristic trend of the maintainability as function of the voltage level, valid for all the MRs. For instance, if on one hand for Torino the maintainability decreases when considering OHTLs at higher voltage levels, suggesting that MTTR increases with voltage level as the complexity of equipment is going up, the opposite situation occurs for MR Roma for which the maintainability increases as the voltage level increases. This highlights the strong dependence of the maintainability on the geographical area of interest and the operating voltage of the OHTLs as the typology of supplied loads and energy demand may change drastically. Furthermore, each region has its own policy and environmental conditions that have their impact on the maintenance management.

\section{CONCLUSIONS}

In this paper, an analysis of the outages occurred to the Italian Overhead Transmission Lines from 2008 to 2015 has been carried out. In particular, two different analyses, one based on a new reliability index, namely the Severity Factor, and one based on a traditional RAM technique, have been performed, focusing on the geographical distribution of the OHTLs. The Severity Factor has been introduced in order to provide a useful tool for the identification of the most critical OHTLs affecting and preventing the optimal operation of the national transmission system. The main differences with respect to other transmission systems reliability metrics found in the literature have been highlighted. The evaluation of the SF has shown that the impact of the outages on the OHTLs reliability is generally not uniform across the country but depends on the considered

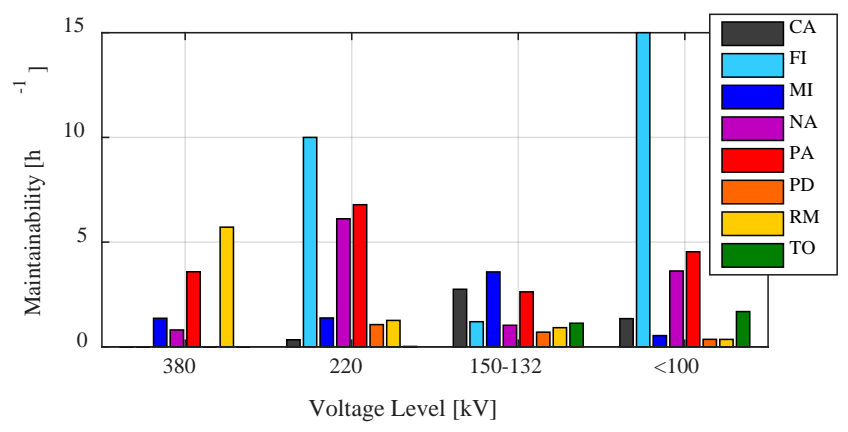

Figure 6. Maintainability for different voltage subpopulations and macro regions. 
region. Further, for each analysed region, the voltage levels more prone to failure have been determined. The same analysis has been carried out also at a national level and the results have shown that the situation depicted at such level is not necessarily replicated at the regional level, justifying the classification of outages data according to regional criteria, as proposed in this contribution. For what concerns the RAM analysis, it has been performed starting from the same outage data. The most important conclusion that can be derived is that the joint evaluation of the results obtained from these two different approaches provides a set of information that enables a complete evaluation about the impact of outages on the OHTLs operation, involving at the same time key concepts of the reliability theory as MTTR, MTBF, availability, failure rate and maintainability but also other physical and economical concepts as interrupted power and not- transmitted energy. It can be stated, therefore, that the proposed methodology is a useful and effective tool for the identification of the transmission network criticalities and the prioritization of the research activities aimed at a better understanding of the failure modes and failure mechanisms affecting the lines, with the final objective to increase their reliability and enhance the maintenance activities planning and performances, at both local and national levels.

\section{REFERENCES}

[1] T. Gonen, "Electrical Power Transmission System Engineering: Analysis and Design", CRC press, Third edition, 2014.

[2] J.C. Pohlman, K.E. Lindsey, R. F. Corpuz, "Controlling the economic risk from catastrophic failure of overhead transmission lines", IEEE ESMO, 1998, pp. 300-310.

[3] D. O. Koval, A. A. Chowdhury, "Assessment of TransmissionLine Common-Mode, Station-Originated, and Fault-Type Forced-Outage Rates", IEEE Transactions on Industry Applications, vol. 46 , no.1, Jan. - Feb. 2010, pp.313-318.

[4] B. Shen, D. Koval, W. Xu, J. Salmon, S. Shen, "An analysis of extreme-weather-related transmission line outages", IEEE Canadian Conference on Electrical and Computer Engineering, vol.2, pp.697-700, vol.2, 24-28 May 1998.
[5] D. Koval, B. Shen, S. Shen, A. A. Chowdhury, "Modeling Severe Weather Related High Voltage Transmission Line Forced Outages", in IEEE Transmission and Distribution Conference and Exhibition, 2006, pp.788-793.

[6] Y. Cheng, C. Niu, C. Li, "The reliability evaluation method of high voltage overhead transmission lines", International Conference on Condition Monitoring and Diagnosis, 2008, pp. 566-569.

[7] I. Dobson, B. A. Carreras, "Number and propagation of line outages in cascading events in electric power transmission systems", 48th Annual Allerton Conference on Communication, Control, and Computing, 2010, pp.1645-1650.

[8] M. Faifer, M. Khalil, C. Laurano, G. Leone, S. Toscani, "Outage Data Analysis and RAMS Evaluation of the Italian Overhead Transmission Lines", IEEE International Energy Conference and Exhibition (ENERGYCON), 2016.

[9] C. Laurano, G. Leone, M. Zanoni, "Outage Severity Analysis on Italian Overhead Transmission Lines from a Regional Perspective", 14th IMEKO TC10 Workshop Technical Diagnostics "New Perspectives in Measurements, Tools and Techniques for system's reliability, maintainability and safety", Milan, Italy, June 27-28, 2016.

[10] Rete Elettrica Nazionale, Annual reports of TERNA [Online].Available: http://www.terna.it/default/home_en/electric_system/statistica 1_data.aspx

[11] IEEE std. 493-1990, "IEEE recommended practice for the design of reliabile industrial and commercial power systems", 1991.

[12] IEEE std. 1366-2012, "IEEE guide for electric power distribution reliability indices", 2012.

[13] R. Billinton, R. N. Allan, "Reliability assessment of large electric power systems", 1st edition, Kluwer Academic Publishers, 1988.

[14] H. Pham, "Handbook of Reliability Engineering", Springer 2003.

[15] R. Billinton, R.N Allan, "Reliability evaluation of engineering systems", 2nd edition, Plenum Press, 1996.

[16] B. S. Dhillon, "Maintainability, Maintenance, and Reliability for engineers", CRC Press, 2006.

[17] A. Birolini, "Reliability Enghineering: Theory and Practice", 5 ${ }^{\text {th }}$ edition, Springer, 2007.

[18] R. F. Stapelberg: "Handbook of Reliability, Availability, Maintainability and Safety in Engineering Design", Springer, 2009. 